\title{
Identifying synchronization between flow boiling inside two parallel minichannels using joint recurrence plots
}

\author{
Hubert Grzybowski*, Iwona Gruszczyńska, and Romuald Mosdorf \\ Department of Mechanics and Applied Computer Science, Faculty of Mechanical Engineering, Bialystok University of Technology, \\ Wiejska 45 C, 15-351 Bialystok, Poland
}

\begin{abstract}
In this study the flow boiling inside two parallel tubes with a diameter of $1 \mathrm{~mm}$ was analysed in order to determine synchronization level between the channels. An experimental setup was built to investigate the pressure and temperature oscillation in parallel minichannels. During the experiments, the two-phase flow patterns were recorded by high speed camera and also the presence of vapour in channel outlet was measured by laser-phototransistor sensor. Various types of two-phase flow instabilities were observed in investigated the system. Experiment was carried out for various heat rate and mass flux. The method of identifying synchronization between flow boiling between parallel channel will be presented on measurement data recorded for heat rate $q$ equal to $50.15 \mathrm{~W}$ and the average mass flux $\dot{m}$ equal to $38.8 \mathrm{~kg} / \mathrm{m}^{2} \mathrm{~s}$. The signal was subjected to a nonlinear analysis based on the joint recurrence plot (JRP) method. The JRP method was carried out in order to determine synchronization level between signals from parallel channels. Results of pressure and laser-phototransistor oscillation analysis shows that during flow boiling phase synchronization and phase shift between the channels can be detected using appropriate $R Q A$ indicators.
\end{abstract}

\section{Nomenclature}

JR joint recurrence matrix

JRP joint recurrence plot

$l \quad$ length of diagonal line

$L_{\max } \quad$ length of the longest diagonal line

$m$ embedding dimension

$\dot{m} \quad$ mass flux $\left(\mathrm{kg} \cdot \mathrm{m}^{-2} \cdot \mathrm{s}^{-1}\right)$

$n \quad$ embedding dimension

$N \quad$ number of samples

$p \quad$ pressure $(\mathrm{Pa})$

$q \quad$ heat rate $(\mathrm{W})$

PS phase synchronization

佧 set of real numbers

R recurrence matrix

$\mathrm{RP}$ recurrence plot

$R Q A \quad$ recurrence quantification analysis

$T$ temperature difference $(\mathrm{K})$

$t \quad$ time (s)

$x \quad$ measured value

$y \quad$ measured value

Greek symbols

$\varepsilon \quad$ threshold for $R P$ computation

$\Theta \quad$ Heaviside function

$\tau \quad$ time delay (samples)

Subscripts

1,2 number of channel

$i, j \quad$ indices

\section{Introduction}

Two-phase flow boiling instabilities can occur in any heat exchanging system equipped in small diameter channels [1]. This makes it difficult to precisely determine the optimal operating conditions of the systems. Some flow oscillations may cause undesirable effects such as mechanical vibrations, burn-out and thermal fatigue. Instabilities are associated with formation of different flow patterns and also leads to formation of various pressure drop and wall temperature oscillations [2]. The oscillations caused by one type of instability are superimposed to another type which may lead to chaotic oscillations [3]. In parallel minichannels also flow maldistribution phenomenon took place [4]. All those effects reduce heat transfer effectiveness. This paper presents a method of identifying synchronization between flow boiling between two parallel minichannels system. The pressure and temperature fluctuations occurring during flow boiling in two parallel minichannels with diameter of $1 \mathrm{~mm}$ have been experimentally investigated and analysed. Data acquired for constant heat rate $50.15 \mathrm{~W}$ average mass flux $\dot{m}$ equal to $38.8 \mathrm{~kg} / \mathrm{m}^{2} \mathrm{~s}$ was taken under consideration. The joint recurrence plot (JRP) method was used to measure synchronization level between signals from each

\footnotetext{
Corresponding author: h.grzybowski@pb.edu.pl
} 


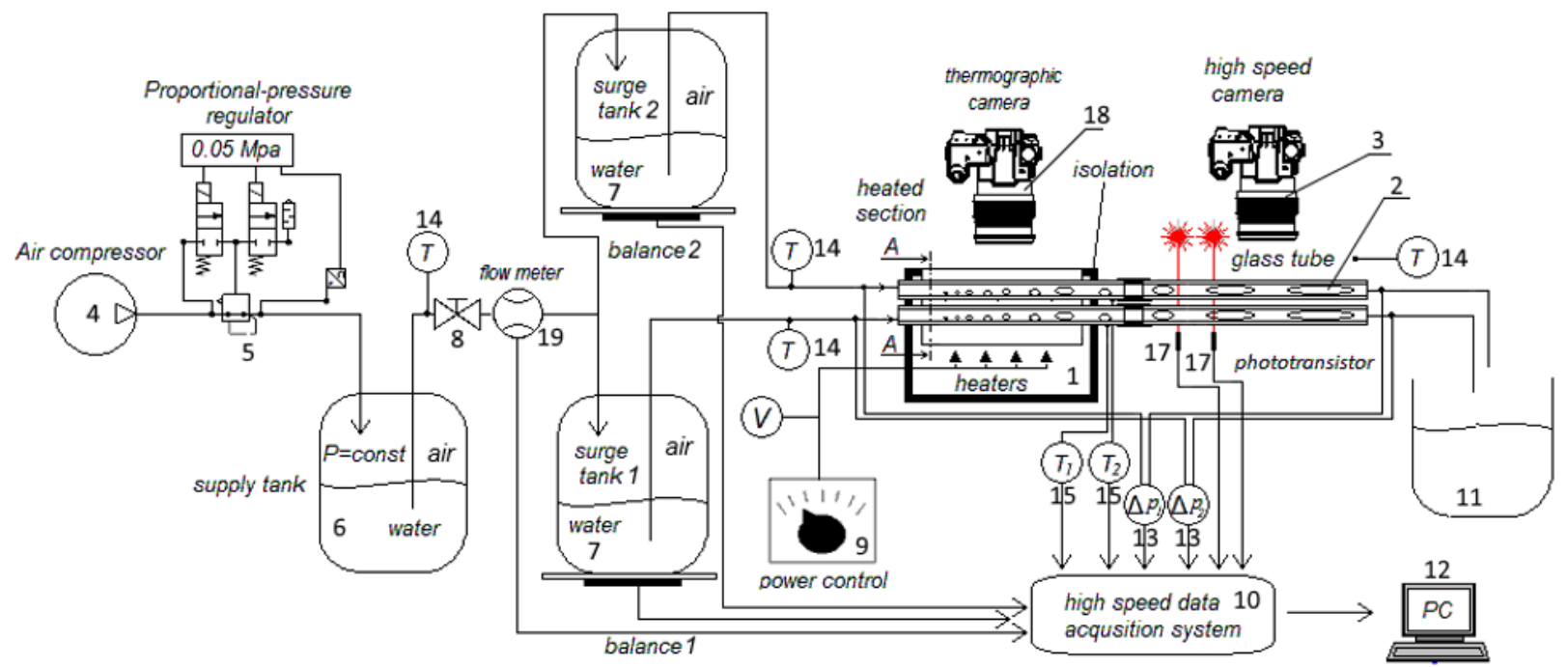

Fig. 1. Scheme of the experimental setup.

channel. Detection of phase synchronization (PS) by means of recurrences was done by calculation of recurrence rate directly from the RP. The $1 / L_{\max }$ indicator for laser-phototransistor series was used to reveal moments when pressure fluctuations are shifted relatively to each other.

\section{Experimental setup}

In this study the flow boiling inside two parallel tubes with a diameter of $1 \mathrm{~mm}$ was analysed. The experimental procedures were carried out for three different types of liquid supply system. Fig. 1 shows a diagram of the experimental setup in one of three liquid supply configurations. Distilled water (working fluid) has been pushed out of the supply tank $(6-$ Fig. 1$)$ by means of the compressed air. A proportional pressure regulator (5 - Fig. 1) was used to maintain a constant overpressure of $15 \mathrm{kPa}$ in the supply tank. A ball valve $(8-$ Fig. 1$)$ was installed between two surge tanks ( 7 - Fig. 1) and the supply tank (6 - Fig. 1), where it was possible to measure and regulate the liquid flow rate.

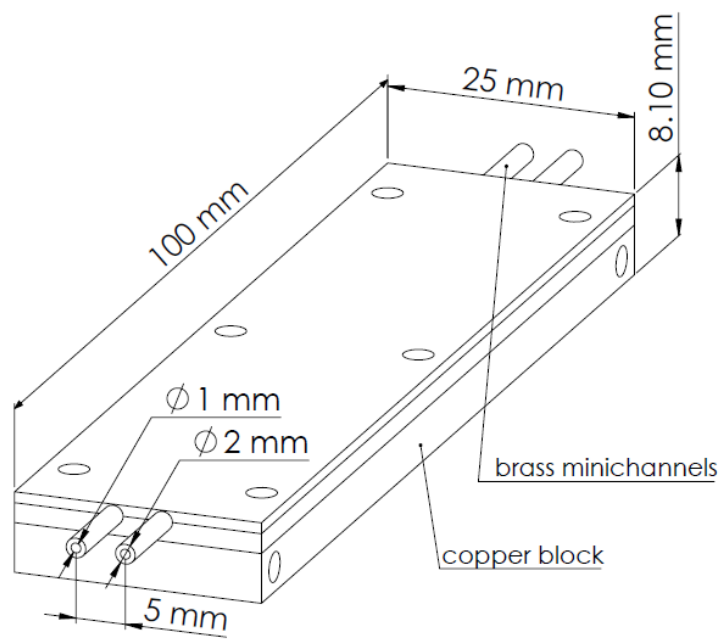

Fig. 2. Schematic diagram of test section.
The water flow starting in the supply tank goes to two separate surge tanks and then goes to a heated section (1 - Fig. 1) consisting of two circular brass minichannels with an inner diameter of $1 \mathrm{~mm}$, an outer diameter of $2 \mathrm{~mm}$ and a length of $150 \mathrm{~mm}$. The minichannels were installed in the copper block with dimensions of $25 \times 8.1 \times 100 \mathrm{~mm}$, shown in Fig 2 . The copper block was electrically heated and isolated by ceramic fibre wool. The heat was supplied to the pipes on a length of $100 \mathrm{~mm}$. The pressure difference in separate channels was measured using the silicon pressure drop sensors MPX50DP with a response time of $0.001 \mathrm{~s}$. The outlet of brass pipes (length $25 \mathrm{~mm}$ ) was isolated and the thermocouples (type $\mathrm{K}$ with diameter of $0.081 \mathrm{~mm}$ and $\pm 0.8 \mathrm{~K}$ accuracy, response time $0.025 \mathrm{~s}$ ), was placed in the distance of $10 \mathrm{~mm}$ from the pipe outlets. The amount of vapour flowing through the glass pipes was measured by laser-phototransistor sensors placed $20 \mathrm{~mm}$ downstream from minichannels outlets. During the experiment, the flow patterns inside the glass pipes were recorded using a high speed camera. The mass flux of water was measured by flow meter calculating the mass increment per unit time. All data was collected by a data acquisition system with a sampling frequency of $1 \mathrm{kHz}$. During the experiment various types of two-phase instabilities were observed. Similar experiment was conducted in our previous work [5] on similar single channel experimental setup.

\section{Characteristics of recorded signals}

Experimental measurements for parallel channels ware carried out at a constant amount of heat rate generated by heaters $50.15 \mathrm{~W}$. The average mass flux $\dot{m}$ was computed from value recorded by flow meter divided by cross-sectional area of both channels. Thus, the average mass flux was equal to $38.8 \mathrm{~kg} / \mathrm{m}^{2} \mathrm{~s}$ and oscillated in range $\pm 0.5 \mathrm{~kg} / \mathrm{m} 2 \mathrm{~s}$ due to long periodic pressure drop oscillations. Pressure drop was measured by differential pressure drop sensor with a response time of $0.001 \mathrm{~s}$. The content of the minichannel (vapour or liquid) has been qualitatively assessed using the laserphototransistor sensors (17-Fig.1). When the channel is 
filled with water, the sensor generates a high voltage signal (above $3 \mathrm{~V}$ ). When the bubble or slug occurs inside the minichannel, then the sensor generates a low voltage signal (below $1 \mathrm{~V}$ ). Above properties of laserphototransistor sensors allow using to use the signal from sensors for qualitative assessment of the presence of bubbles or slugs in the minichannel. Fig. 3 shows input signal conversion to dash plot which will be used in next figures.

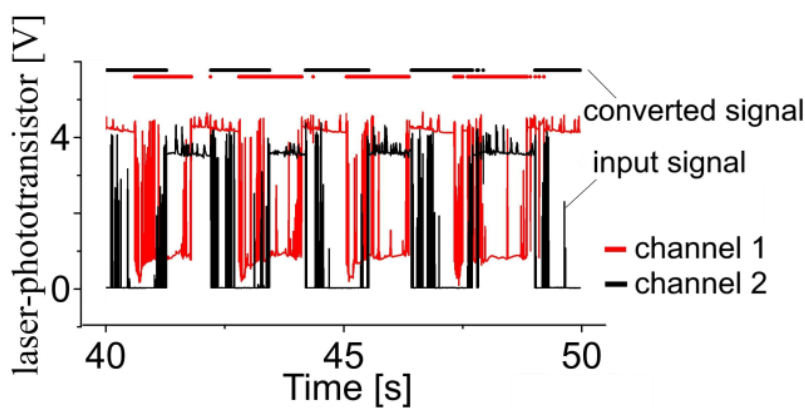

Fig. 3. Laser-phototransistor signal conversion to dash plot.

\section{Synchronization analysis method}

The times series measured for each channel can be treated as two dynamical system with mutual interaction. The channels can be synchronized by means of thermal or pressure effects. Joint recurrence plot (JRP) is a technique used to visualize recursive states occurring in one dynamic system with happening at the same time in another dynamic system. JRP is an RP extension that refers to only one system. The JRP developed by Romano [6] is associated with the creation of graph which shows times at which a recurrences in one dynamical system occurs in another dynamical system and can be used to detect phase synchronization. Obtained recurrence plots are based on information on the reconstruction of trajectories of attractors from time series using the delay method. This method assumes that the coordinates of the attractor points are calculated based on the order of the samples between which the distance equals of the time delay. Method was performed for the signals obtained from the pressure sensors and laser-phototransistors. However, the JRP and RPs were obtained for selected time windows from all signals containing 5000 samples. Values of time delay, embedding dimension and threshold were chosen for two time series, the gained JRP was the result of the multiplication of RPs (eq. 1,2) according to the formula (eq. 3):

$$
\begin{gathered}
\mathbf{R}_{\mathrm{i}, \mathrm{j}}=\theta\left(\varepsilon_{x}-\left\|x_{i}-x_{j}\right\|, x_{i} \in \mathbb{R}^{m}, i, j-1, \ldots, N,\right. \\
\mathbf{R}_{\mathrm{i}, \mathrm{j}}=\theta\left(\varepsilon_{y}-\left\|y_{i}-y_{j}\right\|, y_{i} \in \mathbb{R}^{n}, i, j-1, \ldots, N,\right. \\
\mathbf{J R}_{\mathrm{i}, \mathrm{j}}=\theta\left(\varepsilon_{x}-\left\|x_{i}-x_{j}\right\|\right) \cdot \theta\left(\varepsilon_{y}-\left\|y_{i}-y_{j}\right\|\right) \\
x_{i} \in \mathbb{R}^{m}, y_{i} \in \mathbb{R}^{n}, i, j-1 \ldots N,
\end{gathered}
$$

where: $N$ is a number of considered states $x_{i}, \varepsilon$ is a threshold distance with $m$ (or $n$ ) - dimensional phase space, $\|-\|$ - norm, $\theta(\cdot)$ - Heaviside step function.

The mutual information method [7] was used to determine the time delay for the reconstruction attractors. Time delay for which the mutual information obtained first minimum is the correct value of $\tau$. To determine the proper embedding dimension of the attractors in phase space was chosen the algorithm of the Nearest False Neighbor [8]. The main idea of this method is to see how the number of adjacent points in the immersion space varies with the increase embedding dimension. Proper embedding dimension for attractor reconstruction is defined as the dimension for which the fraction of false points reaches zero. The threshold $(\varepsilon)$ can be set as a fixed or selected value to get the overall percentage of recursive points on the recursive graph (ie. to obtain the assumed density graph). In this case, $\varepsilon$ was determined by the size of the reconstructed attractor and should be equal to $20 \%$ of the attractor diameter in $\mathrm{m}$ dimensional phase space. The threshold value is associated with the number of black dots on the RP. When this value is too large, the RP becomes too black, whereas when it is low, too little information about system dynamics is provided.

Joint recurrence quantification analysis $(J R Q A)$ is a method for quantitative analysis of characteristic structures of overlapping recurrence graphs of two dynamic systems. JRQA is an extension of the $R Q A$ method, a tool proposed by Zbilut and Webber [9], which provides additional information about the recurrence graph of a single time series. Therefore $J R Q A$ generates the indicators which used to describe the joint dynamics of two time series. With the $13 R Q A$ measures are described and developed by Marwan et al. [10]. For the analysis of this paper $R R$ (Recurrence rate) and $L_{\max }$ (longest diagonal line) indicators were selected. The number of points in the JRP can be quantified by the recurrence rate, $R R$, which is a ratio of recurrence points to all possible recurrence points in the RP. It is defined as follows [6]:

$$
R R=\frac{1}{N^{2}} \sum_{i, j=1}^{N} R_{i, j}
$$

Second indicator $L_{\max }$ is defined as the time in which the state does not change or change very slowly:

$$
L_{\max }=\max \left(\left\{l_{i} ; i=1, \ldots, N_{l}\right\}\right)
$$

The calculations have been made using the CRP toolbox for Matlab ${ }^{\circledR}[11]$.

\section{Results and discussion}

Fig. 4 presents pressure, temperature and laserphototransistor time series in comparison with $J R Q A$ analysis results. 


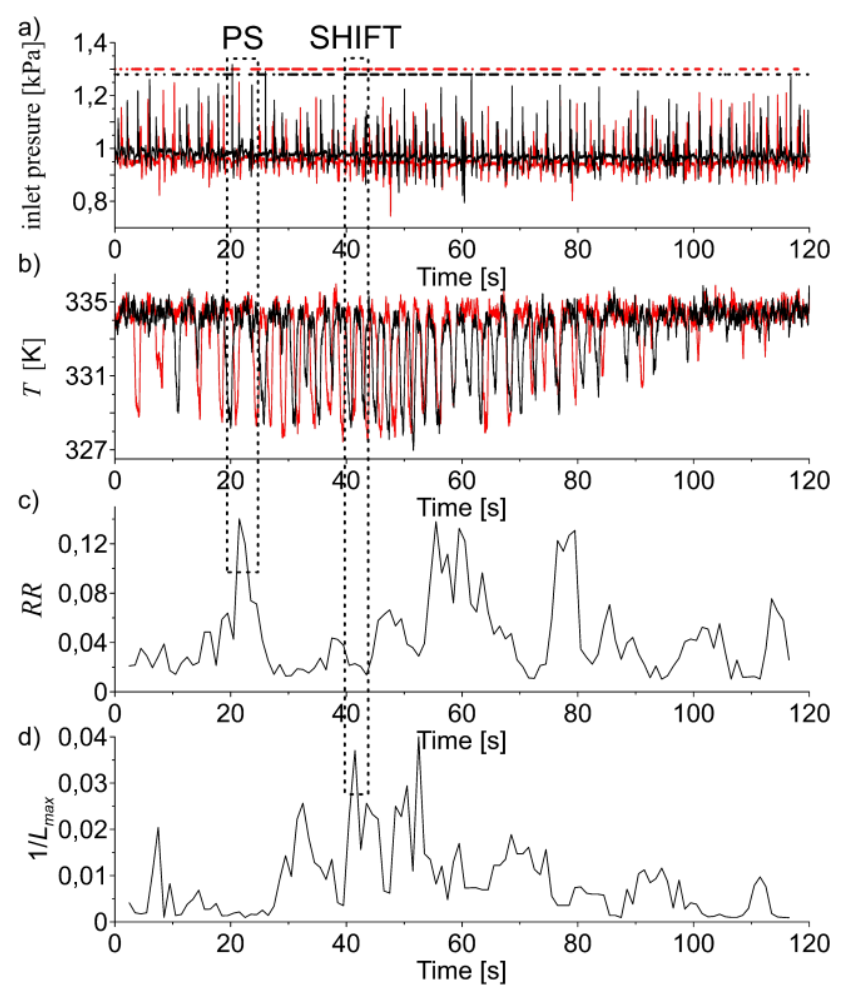

Fig. 4. Inlet pressure and temperature oscillations in comparison with analysis results.

Time series data acquired from first channel was marked in red color and for the second channel was black. The thermocouples time series graphs (Fig. 4a) shows that boiling in each channels causes periodic temperature oscillations, which can be shifted, synchronized or desynchronized. In addition, different flow patterns were observed in each channel (Fig.5).

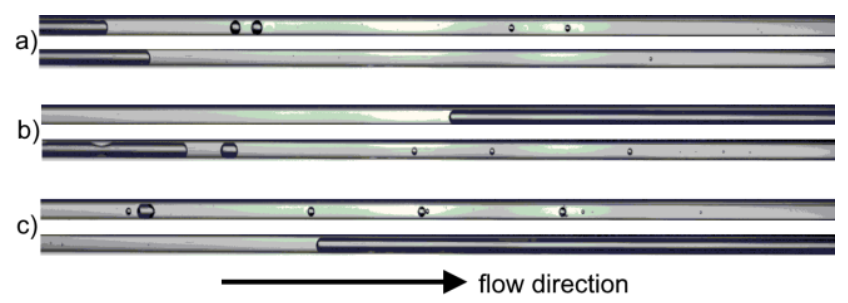

Fig. 5. Flow patterns recorded at the same time in both channels.

Mainly slug or bubbly flows with temporary flow reversal were observed. Due to the chaotic nature of time series associated with flow boiling there is hard to distinguish degree of synchronization between the channels. Therefore, an attempt was made to determine the degree of synchronization based on analysis of pressure and laser-phototransistor time series. The Fig.4c shows $R R$ changes for pressure signals. Detection of phase synchronization (PS) by means of recurrences is done by calculation of recurrence rate directly from the RP. The method can detect PS even in time series which are strongly corrupted by noise [12]. According to the analyzed signals, high values of RR reveals moments when pressure peaks occurs at the same time in both channels. Example of PS $(R R>0.1)$ was marked with dashed line rectangle in Fig.4.

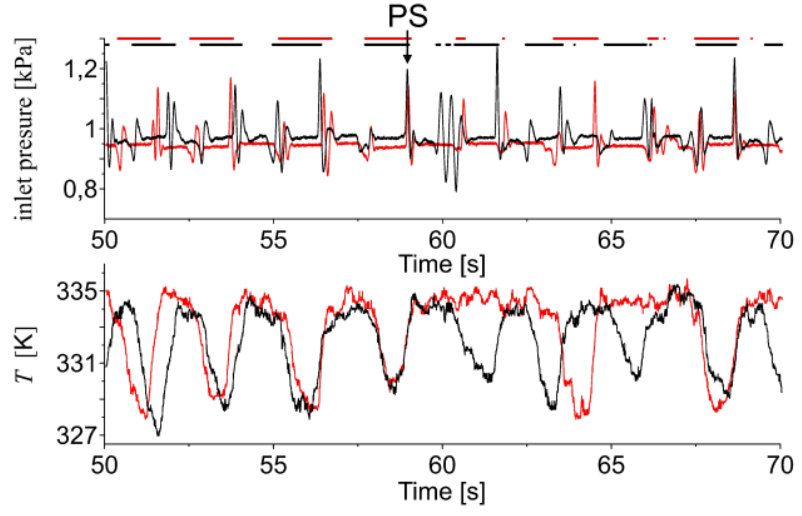

Fig. 6. Inlet pressure and temperature time series for which the moment of phase synchronization can be observed.

For the analyzed series, also the time interval between 58-60 s had the high value of the RR indicator. The part of the signals for which the moment of phase synchronization $(R R>0.1)$ is clearly marked is shown in the Fig.6. It can be also observed form temperature times series that before PS temperature fluctuation also tends to synchronization but after moment of pressure peak synchronization moment there is a temperature loss of synchronization.

Fig.5a presents the flow pattern observed when phase synchronization occurs. At the same time from both channel flows out vapour slugs. Another parameter that was considered during the analysis was $L_{\max }$. This indicator is related with the positive Lyapunov exponents [13], which is a measure of the chaos in system. Rapid changes of this indicator detects chaos to periodic chaos transitions [6]. It was observed that high values of $1 / L_{\max }$ indicator for laser-phototransistor series reveals moments when pressure fluctuations are shifted relatively to each other or more precisely the moment in which pressure in one channel oscillates and in the second it does not. Two of those moments $\left(1 / L_{\max }>0.02\right)$ were marked with dashed line rectangle in Fig.7. Dependence between pressure oscillation shift and temperature increase can be observed. When pressure oscillations occur in one channel at the same time the temperature increases and then the same phenomenon with a certain shift is repeated in the second channel. Fig.5b and Fig.5c presents the flow pattern observed when phase shift occurs. In Fig.5b vapour slug already flowed out from the upper channel and in the bottom channel is just forming. Next in Fig.5c slugs flowing in bottom channel can be observed. 


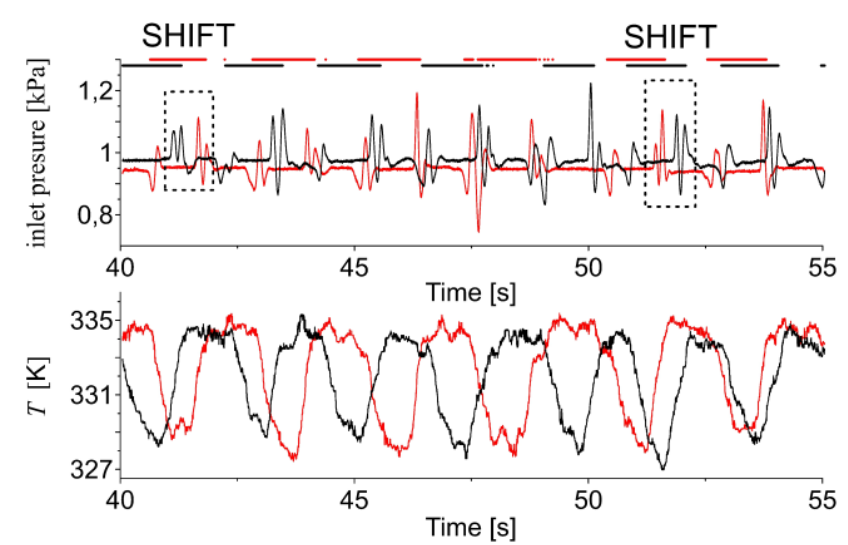

Fig. 7. Inlet pressure and temperature time series for which the moment of phase shift can be observed.

\section{Conclusions}

In this study the flow boiling inside two parallel tubes with a diameter of $1 \mathrm{~mm}$ was analysed in order to determine synchronization level between the channels. Due to the chaotic nature of time series associated with flow boiling it is hard to distinguish degree of synchronization between the channels. The JRP method was used to detect phase synchronization and phase shift between signals from each channel. Recurrence rate indicator was used in order to detect moment of phase synchronization and $1 / L_{\max }$ indicator was used to detect phase shift between the signals. It was observed that before PS temperature fluctuation also tends to synchronization but after the pressure peak synchronization moment there is a temperature loss of synchronization. Also dependence between pressure oscillation shift and temperature increases in both channel was observed. The recordings from high speed camera confirmed the areas detected by JRP analysis.

\section{Acknowledgements}

The study has been accomplished under the research project UMO-2015/17/N/ST8/03079 financed by the Polish National Science Centre.

\section{References}

1. T. G. Karayiannis, M. M. Mahmoud, Appl. Therm. Eng. 115, 1372-1397 (2017)

2. L. C. Ruspini, C. P. Marcel, A. Clausse, Int. J. Heat Mass Transf. 71, 521-548 (2014)

3. Chin-Jang Chang, R. T. Lahey, Nucl. Eng. Des. 167, 307-334 (1997)

4. E. R. Dario, L. Tadrist, J. L. G. Oliveira, J. C. Passos, Appl. Therm. Eng. 91, 924-937 (2015)

5. H. Grzybowski, R. Mosdorf, Int. J. Heat Mass Transf. 73, 500-510 (2014)

6. N. Marwan, M. Carmen Romano, M. Thiel, J. Kurths, Phys. Rep. 438, 237-329 (2007)
7. M. S. Roulston, Phys. Nonlinear Phenom. 125, 285294 (1999)

8. M. B. Kennel, R. Brown, H. D. I. Abarbanel, Phys. Rev. A 45, 3403-3411 (1992)

9. J. P. Zbilut, A. Giuliani, C. L. Webber, Phys. Lett. A 246, 122-128 (1998)

10. N. Marwan, N. Wessel, U. Meyerfeldt, A. Schirdewan, J. Kurths, Phys Rev E 66, 026702 (2002)

11. N. Marwan, Cross Recurrence Plot Toolbox for MATLAB®, Ver. 5.21 (R31c), http://tocsy.pikpotsdam.de/CRPtoolbox/, accessed 2017-01-02

12. M. C. Romano, M. Thiel, J. Kurths, I. Z. Kiss, J. L. Hudson, EPL Europhys. Lett. 71, 466 (2005)

13. L. L. Trulla, A. Giuliani, J. P. Zbilut, C. L. Webber, RPhys. Lett. A 223, 255-260 (1996). 